\title{
Características socio-económicas de las familias de mujeres víctimas de violencia de género con hijos/hijas testigos, que asisten al servicio comunitario Casa de la Mujer Bañado Tacumbú, 2020
}

\section{Socio-economic characteristics of the families of women victims of gender violence with witness sons / daughters, who attend the Casa de la Mujer Bañado Tacumbú community service, 2020}

\author{
Analía Roxana Carosini Miranda ${ }^{a}$ Lilian Paola Morales Ruiz ${ }^{a}$
}

\begin{abstract}
Resumen
Es un estudio exploratorio de enfoque cualitativo que permite comprender los relatos de experiencias de las mujeres, desde una mirada con enfoque de género y derechos humanos. Se desarrolló un análisis crítico en torno a dimensiones como ser: estructura y relacionamiento familiar, condiciones económicas, educativas, de salud y finalmente las expresiones de violencia de género. Entre los principales hallazgos, el estudio muestra que las mujeres entrevistadas provienen de familias nucleares, durante su niñez y adolescencia todas han sido testigos de violencia de género ya que ésta era ejercida por parte de sus padres hacia sus madres. Han sido trabajadoras adolescentes, priorizaron el sustento económico diario y en esta etapa abandonaron o fueron privadas de la formación académica; acceden a servicio de salud públicos. Tuvieron como primer cónyuge al padre de sus hijos e hijas, que pasado el tiempo a su vez se convirtieron en sus principales agresores. Las mujeres se encontraron dentro del círculo de violencia por más de diez (10) años, durante todo este tiempo sus hijos e hijas fueron testigos de tal realidad. La Casa de la Mujer, servicio comunitario, ha sido clave en la transformación cultural de estas mujeres al momento de romper el círculo de violencia y denunciaron tal situación, fueron acompañadas psicológica y legalmente sintiéndose como sujetos de derecho y reconociendo la posibilidad de una vida libre de violencia.
\end{abstract}

Palabras clave: género, violencia de género, testigos de violencia, familia, perspectiva de género. a Universidad Nacional de Asunción, Facultad de Ciencias Sociales, Paraguay.

Correspondencia a: acaromira@gmail.com

Recibido:

1o de setiembre de 2020 Aceptado:

14 de noviembre de 2020

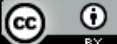

Artículo publicado en acceso abierto bajo la Licencia Creative Commons.

Cita:

Carosini Miranda, A. A., \& Morales Ruiz, L. P. Características socioeconómicas de las familias de mujeres víctimas de violencia de género con hijos/ hijas testigos, que asisten al servicio comunitario Casa de la Mujer Bañado Tacumbú, 2020. (2020). Kera Yvoty: reflexiones sobre la cuestión social, 5, 112-123. 


\begin{abstract}
It is an exploratory study with a qualitative approach that allows us to understand the stories of women's experiences, from a perspective with a gender and human rights perspective. A critical analysis was developed around dimensions such as: family structure and relationship, economic, educational, health conditions and finally the expressions of gender violence. Among the main findings, the study shows that the women interviewed come from nuclear families, during their childhood and adolescence all have witnessed gender violence since it was exercised by their fathers towards their mothers. They have been adolescent workers, they prioritized daily economic support and at this stage they abandoned or were deprived of academic training; they access public health services. They had as their first spouse the father of their sons and daughters, who over time in turn became their main aggressors. Women were within the circle of violence for more than ten (10) years, during all this time their sons and daughters were witnesses of such a reality. La Casa de la Mujer, community service, has been key in the cultural transformation of these women at the time of breaking the cycle of violence and denounced such situation, they were psychologically and legally accompanied, feeling as subjects of law and recognizing the possibility of a free life of violence.
\end{abstract}

Keywords: gender, gender violence, witnesses of violence, family, gender perspective.

\section{Introducción}

En este estudio al aludir a la violencia de género se hace referencia a la violencia ejercida contra la mujerenelámbito familiar, donde el agresor directo es o fue el esposo, ex esposo, concubino, ex concubino, novio, ex novio, ya que las estadísticas mostraron que son ellos los principales agresores.

En los años 2014 y 2015, según ONU Mujeres (2016) entre el $76 \%$ y el $78 \%$ de las mujeres que acudieron al Servicio de Atención a la Mujer (SEDAMUR), dependiente del Ministerio de la Mujer, indicaron que la violencia provino de su esposo, concubino o ex concubino, es decir, ocurre en el marco de relaciones de pareja. El mayor índice de violencia, según la misma fuente, proviene de los concubinos que en el 2014 indica (38\%), en el 2015 (37\%), seguidos del esposo (22\%) y (23\%) y luego el ex concubino (18\%) y (16\%).

Si bien la violencia hacia las mujeres constituye un fenómeno amplio de violaciones a sus derechos, esta misma problemática social trae consigo la vulneración de derechos de otras personas. En este sentido se habla de la presencia de los hijos e hijas dentro de este entorno familiar, quienes se encuentran en condición de testigos de violencia.
La violencia contra la mujer es una violencia estructural presente en la división y el ejercicio del poder político, en el acceso a los recursos económicos, en la división del trabajo productivo y en las tareas del hogar y ciertamente en el ejercicio de la violencia en sus diferentes manifestaciones en el ámbito familiar y relaciones de pareja.

En este sentido Segovia (2019, en comunicación personal) psicóloga y orientadora del Ministerio de la Niñez y Adolescencia sostiene que:

Esta situación de violencia genera un montón nuevamente (sic.) de consecuencias directa en la vida de los niños; económica, sociales, de salud física, de salud mental, dejan de ir a la escuela, afecta en todo lo que es su vida, en las relaciones con sus pares porque van y repiten nuevamente.

Existen escasos datos estadísticos sobre testigos de violencia, sin embargo, por la cantidad de niños/as que fueron atendidos en el albergue Casa Abrigo "Mercedes Sandoval" para víctimas de violencia intrafamiliar dependiente del Ministerio de la Mujer se pudo tener una 
Carosini Miranda, A. A., \& Morales Ruiz, L. P. Características socio-económicas de las familias de mujeres víctimas de violencia de género con hijos/hijas testigos, Bañado Tacumbú, 2020.

aproximación de cuántos niños o niñas han estado expuestos a un entorno de violencia.

Otro dato relevante es el que mostró un estudio realizado en el Hospital de Clínicas, Cátedra de Psiquiatría, en el cual fueron incluidos 101 pacientes consultantes entre el año 2010 al 2013 con una franja de edad de 3 a 18 años, el cual 53,5\% ( $n=54)$ del total había sido testigo de violencia entre padres (Arrom, 2015).

De la misma forma, la OMS (2016) argumenta que la exposición a la violencia de pareja también se incluye entre las formas de maltrato infantil y sostiene que estas víctimas en su vida adulta corren mayor riesgo de sufrir problemas conductuales, físicos, mentales como: depresión, consumo de tabaco, actos de violencia (como víctimas o perpetradores), obesidad, adicción al alcohol o drogas, comportamientos sexuales de alto riesgo, embarazos no deseados.

Esas consecuencias evidencian que los niños, niñas y adolescentes se encuentran en total vulneración de sus derechos al ser parte de este contexto de violencia, entendiendo que según UNICEF (2012) "los niños no son víctimas solo cuando la violencia se dirige contra ellos, sino también cuando viven la violencia cotidiana, cuando son testigos, cuando se lástima a quienes ellos quieren" (p. 105).

Estas consideraciones destacan que las consecuencias a corto plazo se pueden dar a nivel cognitivo, emocional, conductual en niños, niñas y adolescentes; como así también existe mayor predisposición a sufrir limitaciones sociales, emocionales, conductuales, cognitivas durante toda la vida; a adoptar comportamientos de riesgo para la salud y la vida, llegando a afectar su funcionamiento en la vida adulta.

Finalmente, se suma a las situaciones de violencia de género, las condiciones socio-económicas de las mujeres. Aunque la violencia de género se pueda dar independientemente al nivel económico, educativo o social, la condición de ser mujery pertenecer a un sector popular caracterizada por la vulnerabilidad económica determina una forma diferente la garantía y protección de los derechos humanos, ya que la pobreza también se relaciona con una imposibilidad para acceder a ciertos servicios públicos (Dobrée, 2018). Es en este sentido, que se plantea las siguientes interrogantes sobre las particularidades de cómo afecta a las mujeres de sectores populares.

a) ¿Cuáles son las características socio-económicas de familias de mujeres víctimas de violencia de género con hijos e hijas testigos, que asisten al servicio comunitario Casa de la Mujer Bañado Tacumbú, 2020?

b) ¿Cómo es la estructura y el relacionamiento familiar de mujeres víctimas de violencia de género con hijos e hijas testigos de violencia?

c) ¿Cuáles son las condiciones económicas, educativas y de salud de familias de mujeres víctimas de violencia de género con hijos e hijas testigos de violencia?

d) ¿Cómo se expresa la violencia de género en mujeres con hijos e hijas testigos de violencia?

\section{Materiales y Métodos}

Esta investigación es de tipo cualitativa, exploratoria, descriptiva. La población de estudio está constituida por 21 mujeres que asisten al servicio comunitario Casa de la Mujer Bañado Tacumbú, en el año 2020. Teniendo en cuenta criterios de inclusión y exclusión se abordó a 6 mujeres víctimas de violencia de género con hijos e hijas testigos de violencia de este servicio comunitario. Se utilizó como técnica la entrevista en profundidad ya que permitió recabar datos reales en el campo de estudio que hace referencia a la interacción entre constituciones psicológicas personalesy conductas sociales específicas: agresividad, violencia, entre otros (Betthyany y Cabrera, 2011). Para el análisis se utilizó la información obtenida de las madres (sujeto); interpretada a la luz de los elementos teóricos que sustentan la investigación (marco teórico). Se elaboró un documento de consentimiento 
informado, el cual fue entregado $y$ explicado en detalle a las participantes. También se solicitó su permiso para grabar y tomar notas. El presente documento utiliza nombres ficticios en las citas de las entrevistas realizadas, a fin de garantizar la confidencialidad de la información y la identidad de las participantes y sus hijos e hijas.

\section{Resultados \\ 3.1. Estructura y relacionamiento familiar}

Antecedentes familiares y estructura de la familia

Las mujeres entrevistadas en su mayoría residían en el Barrio Sajonia, luego cuando formaron pareja se trasladaron al Bañado Tacumbú. Dos de ellas hasta su adolescencia residían en el interior del país (Chaco - Concepción).

Las entrevistadas provienen de familias que tienen en promedio de 5 a 11 hermanos. El aspecto resaltante está en que todas las mujeres manifestaron ser testigos de violencia de parte de su papá hacia su mamá.

Algunas expresiones de las mujeres entrevistadas en el presente estudio, testigos de violencia de sus progenitores/as son:

Y yo soy la hermana mayor y yo lo que me hacía cargo de mis hermanos porque pasé también violencia doméstica por parte de mi papá entre mi mamá. (Eo1, 2020)

Yo llegué a ver cuándo mi papá le abofeteó a mi mamá y yo le enfrenté a mi papá y entonces él no supo cómo reaccionar, pero si sufrió mucho mi mamá, todo lo que mi hermano y eso contaban, los mayores, yo soy la última pues, imagínate de los 11 soy la última entonces mi hermano y eso contaban que mi papá era mucho más agresivo anteriormente. (Eo3, 2020).

Todas las mujeres entrevistadas volvieron a ser víctimas en su vida adulta dentro de sus relaciones de pareja, como lo señala (Jelin, 2005), esto refleja una cultura transgeneracional de la violencia hacia las mujeres.

Un hecho que destacar fue lo relatado por una de ellas:

Fui violada por mi padrastro y ahí me enteré que no era parte de ellos. (Eo2, 2020).

Este motivo la llevó a salir de la casa; ya que contó lo sucedido a su mamá adoptiva quien hizo caso omiso al hecho. El Ministerio de la Mujer (2019) recuerda que la violencia se produce sobre la base de la desigualdad, donde lo masculino se considera superior a lo femenino. De esta forma la violencia se transforma en algo habitual, se naturaliza. El agresor permanecerá en silencio y la víctima a su lado, no porque quiere, sino porque no puede salir del ciclo.

Las mujeres entrevistadas, sin embargo, relatan acciones tomadas con respecto a sus parejas e hijas a partir de sus experiencias en su niñez -las que no desean se repita:

Incluso le llegué a meter preso [a mi pareja] en la última vez por intento de violación a nuestra hija, la mayor. (Eo4, 2020)

Yo cuando mi hija le revisaba día y noche sus partes íntimas, a ver si su papá no le tocaba. (Eoz, 2020)

Cinco de seis entrevistadas indicaron que el detonante del maltrato de sus padres hacia sus madres era el alcohol, es decir se debía al estado etílico en el que se encontraban en ese momento.

Tomaba y le pegaba o le quería matar porque mi mamá era liberal y él era colorado y se tomaba de eso. (Eo3, 2020)

Todos los días él tomaba no pasaba un día sin tomar, crecí luego con una trauma yo, porque las veces que tomaba le maltrataba a mi mamá. (Eo6, 2020)

$\mathrm{Al}$ respecto ONU Mujeres (2016) revela que los actos de violencia contra la 
mujer no pueden atribuirse únicamente a factores psicológicos individuales ni a condiciones socioeconómicas como el desempleo. Las explicaciones de la violencia que se centran principalmente en los comportamientos individuales y las historias personales, como el abuso del alcohol o una historia de exposición a la violencia, pasan por alto la incidencia general de la desigualdad de género y la subordinación femenina sistémica. Por consiguiente, los esfuerzos por descubrir los factores que están asociados con la violencia contra la mujer deberían ubicarse en este contexto social más amplio de las relaciones de poder.

Es muy frecuente relacionar el consumo del alcohol con la violencia de género, casi todas las mujeres entrevistadas refirieron que entre su etapa de infancia y adolescencia fueron testigos de violencia, las mismas indicaron que el consumo del alcohol era el detonante de la agresión de sus padres hacia sus madres, lo mismo indicaron hacia la agresión de sus ex parejas. Un día para otro empezaba a tomar, primero era cada sábado, después ya era ya dos veces, después ya continuado, cada tarde, cada noche. $($ E05, 2020).

Las veces que venía borracho, porque él toma nomás, él es así. (Eo1, 2020)

Este señor era muy agresivo, no quería que me vaya [a la escuela para adultos] tomaba mucho también este señor, tomaba luego, y las veces que yo me iba ahí para estudiar, para aprender algo él salía y me gritaba. (Eo6, 2020)

Todas las mujeres entrevistadas, actualmente tienen una vida independiente del lazo que las unía a su madre o padre. Residen en su vivienda propia con sus hijos/as, que en promedio es de 4 a 5 por hogar; con esto se contrasta lo expuesto por Dobreé (2018) quien menciona que la presencia de una considerable población infantil es un dato significativo porque plantea la existencia de una demanda diferenciada de cuidados.

Rol de cada miembro de la familia

Las entrevistadas son jefas de hogar.

La DGEEC (2012) en una de sus publicaciones expresa que las transformaciones en los roles sociales de las mujeres dentro y fuera de la familia han evolucionado de manera muy dinámica: en lo económico, el aumento de su participación laboral y, en lo familiar, el incremento de los hogares encabezados por mujeres.

Se ha destacado la fortaleza del vínculo social entre la madre y los hijos/ hijas. Las madres representan la figura de confianza, protección y afecto para los hijos/hijas.

Yo soy mamá, abuelo, abuela, tía, ere erea para mi nieto ahora. Y para mi familia, que ellos siempre dependen de mí. A veces me dicen: Mariela ${ }^{1}$ tenés que soltarle ya a ellos porque un día de estos vos ya no vas a estar más con ellos. Pero, yo le digo, pero mientras esté con ellos voy a estar porque a mí nunca nadie me apoyó, todas las cosas que a mí me pasó. (E02, 2020)

Lo que a ellos le fortalece siempre es lo que yo les digo verdad; mira, no es fácil la vida, yo no voy a poder mucho tiempo, cosas así voy preparándoles a ellos para que sean personas fuertes. (Е03, 2020)

\section{Rutina cotidiana y relacionamiento familiar}

En el contexto de la pandemia por el Covid-19 hubo modificaciones en la rutina diaria de las mujeres. Mientras que anteriormente los hijos/hijas asistían a sus actividades escolares en turno mañana o doble escolaridad, en este momento todos los integrantes de la familia quedan en la casa, realizando las clases virtuales dentro

1 Los nombres que se utilizan en el presente análisis son ficticios a fin de garantizar la confidencialidad. 
de sus posibilidades.

Ahora por ejemplo cuando vamos hacer la tarea me es muy difícil porque tengo que estar encima de ellos para que hagan. Muchas cosas yo no entiendo, por eso muchas veces le digo a su profesora que muchas veces no hacemos, muchas veces hacemos. Ahora sinceramente están toditos aplazados, hasta el mayor, me peleo todos los días con él para que haga su tarea. (Eo1, 2020).

Y le estamos haciendo sus tareas en cuaderno y lo que es en impresión. Mi nene más grande lo que es un poquito flojo él. (Eo5, 2020)

Conforme fueron pasando las fases del levantamiento gradual del aislamiento preventivo general (cuarentena inteligente), algunas de las entrevistadas retomaron a sus actividades laborales. Muchas de ellas quienes dependen de un trabajo independiente están realizando ollas populares siempre y cuando disponen de insumos donados tanto de instituciones públicas (SEN) como privadas.

Tenía mi olla chica, y venían más criatura, más criatura y así. Siempre empezó de ahí la olla popular. (Eo2, 2020)

Si bien las organizaciones hay que nos ayuda ahora para la olla popular pero esporádico no es diario, la SEN es lo que nos está proveyendo el tema de los insumos pero no para la carne y eso, carne ni verduras, eso nosotros conseguimos así donaciones. (Eo3, 2020)

Dobrée (2020) sobre cuidado ejercido por las mujeres en el contexto Covid 19 expresa que lo cierto es que nos encontramos ante una situación donde la carga de estos trabajos que realizan las mujeres seguramente será mucho mayor como resultado de un evento imprevisto que coloca en riesgo la salud y la vida de amplios sectores de la población.

\section{Condiciones económicas, educativas y de salud \\ 2.1. Condiciones económicas}

Las tareas realizadas por los trabajadores no calificados consisten normalmente en vender mercancías en la calle, lugares públicos o de puerta en puerta; prestar diversos servicios ambulantes; limpiar, lavar y planchar; brindar servicios de portería entre otros.

Algunas de las entrevistadas se dedican a la venta de remedios yuyos, productos de limpieza o trabajo de costura; otras se dedican al servicio de limpieza. Solamente una de ellas cuenta con un salario mensual fijo; las demás perciben de acuerdo a la venta diaria.

Los gastos diarios de la casa cubro con la venta de productos de limpieza, y almorzamos de la olla popular. (Eo1, 2020)

De lo que gano en la costura compro los alimentos. (E03, 2020)

Yo soy mensualera y compró para las cosas de la casa. Día de por medio comemos de la olla popular de Camsat. (E04, 2020)

Todas ellas son sustento económico del hogar, se encargan de los gastos diarios de alimentación; debido a la pandemia Covid 19, los trabajos que realizaban anteriormente se vieron afectados: a una de ellas la empleadora le suspendió temporalmente hasta pasar el plan de levantamiento gradual del aislamiento preventivogeneral (cuarentena inteligente).

\subsection{Condiciones educativas}

En el caso de la presente investigación solo una de las entrevistadas terminó el bachillerato, dos de ellas no fueron escolarizadas y las demás realizaron hasta el zer ciclo de EEB.

Yo no entré en la escuela, hacia el Chaco una semana máximo me fui, después ya no me mandaron. (Eoz, 2020).

Mis hijos másgrandes están en la escuelay yo hice hasta el $8^{\circ}$. (E05, 2020) 
Yo no entré en la escuela porque tenía que trabajar con mi mamá, a veces lloraba le decía yo a mi mamá; ndahaseveima, ndajaposei ha upei oguapyvaera pe che sy oñemoñe'e cheve he'i cheve "che memby ndeñoite ko la che esperanza ha nde manteko la che pytyvõveva" ha aha jey" (Ya no me quiero ir, no quiero hacer. Después se sentaba mi mamá y me hablaba, me decía "mi hija vos sos mi única esperanza y solo vos lo que me ayudas más, entonces me iba otra vez). (Eo6, 2020)

Los hijos e hijas de las entrevistadas se encuentran en periodo de escolarización. Cabe mencionar que una de las hijas es una persona con discapacidad auditiva (Pcd) (sordomuda) y asiste a una escuela inclusiva.

En el país han existido diversos enfoques y abordajes en relación a las personas con discapacidad y con el transcurrir del tiempo se han asumido desafíos en el transitar desde el enfoque de beneficencia hasta el enfoque de derechos humanos, los cuales están reconocidos universalmente (DGEEC, 2012).

Si bien se ha avanzado en el enfoque, una limitante para las personas con discapacidad (Pcd) es la de no poder acceder a este derecho dentro de sus comunidades como es el caso de una de las entrevistadas que debe trasladar a su hija a un barrio aledaño para acceder a la educación inclusiva, lo que implica el desplazamiento de ida y vuelta de la persona.

Pocas de las mujeres y sus hijos pudieron proseguir con sus estudios o realizar cursos de formación.

Algunas expresiones resaltantes relacionadas son:

Yo aprendí a leer en la escuela cuando le llevaba a mis hijos y los números me enseñó mi patrona para poder usar el microondas. (E02, 2020)

Y con mi hija en el brazo tenía que estudiar, no tenía apoyo otra vez al final, tenía que trabajar y estudiar y cuidarle a mi hijo y cuidarle a él también. (E03, 2020)

No quiere luego ni que salga, ni que trabaje; por eso nunca trabajé. (Eo1, 2020)

Según la literatura que los hombres cohíben que las mujeres prosigan sus estudios o se dediquen a otra cosa que no sea las tareas del hogar o el cuidado. (Ministerio de la Mujer, 2013).

\subsection{Condiciones de salud física y emocional}

Las condiciones de salud física de los hijos e hijas presentan afecciones habituales como: alergias y resfríos, dos de ellos se encuentran realizando otro tipo de tratamiento médico.

Uno de los niños presentó complicaciones en el parto, lo que afectó su motricidad fina y gruesa, a consecuencia de eso sigue tratamiento de fisioterapia, que fue suspendido por la pandemia Covid 19.

Una de las niñas tuvo meningitis lo que le dejó con parálisis parcial y acucia profunda. La madre manifiesta que en la escuela recibe talleres de estimulación.

Las mujeres entrevistadas acceden a políticas sociales no contributivas, que como refiere Potyara (1998) son aquellos servicios sociales públicos, total $\mathrm{O}$ parcialmente costeados por el Estado. La población estudiada, las veces que necesita un servicio de salud recurren a los públicos.

Estos servicios generalmente son precarios, están desabastecidos y son limitados; los entes autárquicos, como el Hospital de Clínicas o el Hospital Militar San Jorge de mayor complejidad, tienen un costo y están distantes de la comunidad donde residen las mujeres participantes de este estudio.

Concretamente resultado de episodios de violencia, dos de las madres mencionan que sus hijos/hijas reciben acompañamiento psicológico en la Casa de la Mujer, servicio comunitario, autogestionado que no recibe apoyo estatal pero que representa la contención 
más importante para las mujeres víctimas de violencia y sus hijos e hijas, al ser de rápido acceso por encontrarse dentro de la comunidad.

Se presenta a continuación relato de las madres con situaciones que deben enfrentar con hijos/as adolescentes.

Juancito, hace 3 meses que dejó las drogas, está muy encerrado. Él no es nada agresivo, sino que él no sale de la pieza. Mi hija de 14 años es un poco rebelde, ella hace lo que quiere se va donde ella quiere. (Eo2, 2020).

Y, él es un niño muy dormido, parece que a veces se queda en el aire, así que piensa y vos le hablas y eso lo que yo le llevé un sábado también con el psicólogo y me dice que él no se abre, que él no se quiere abrir. Se le pregunta y dice si - no, no es de eso hablar luego. Se encierra mucho. (E04, 2020).

Además del acompañamiento psicológico la Casa de la Mujer ofrece actividades recreativas como pintata, festivales o charlas los fines de semana para las madres, sus hijos e hijas. Estas actividades están enmarcadas dentro de un programa para erradicar y prevenir la violencia de género en el hogar.

La situación actual también ha empeorado la dinámica familiar donde algún miembro realiza tratamiento médico que han sido suspendidos, lo que dificulta el avance para el bienestar del mismo e implica un trabajo mayor de cuidado para las mujeres.

Altratarsedefamiliasmonoparentales donde la jefatura de hogar recae en las mujeres entrevistadas se percibe que las mismas, parten en peores condiciones para enfrentar esta crisis, presentan una menor participación laboral, les afecta más el desempleo y las responsabilidades de cuidado de niñas, niños y personas con discapacidad, se agudiza.

Las mujeres entrevistadas ya se encontraban en otras condiciones de vulnerabilidad y en estas circunstancias sumamente limitantes de confinamiento o cuarentena, esto se ha agudizado aún más y sus derechos y las de sus hijos e hijas no han sido garantizados.

\section{Expresiones de violencia de género que se dan en mujeres con hijos e hijas testigos de violencia \\ 3.1. Expresiones de violencia de género}

Ejercer violencia es imponer pensamientos o valores con la fuerza, es hacerse valer con el miedo, es no entrar a dialogar, es excluir e infravalorar todo lo que pone en cuestión el poder de quien la pone en marcha y la utiliza (Hernández, 2005).

La bibliografía presenta a la violencia física, como aquella que provoca daño intencional al cuerpo de la víctima, mediante diversas formas entre ellas: puñetazos, patadas, empujones, bofetadas, intentos de ahorcar, tirar del pelo u otra parte del cuerpo, arrastrar por el suelo, mantener atada con cuerdas u otros medios.

Se resalta algunas expresiones de las mujeres sobre la violencia física ejercida hacia ellas:

Y de mi cabello así me estiraba, no era que tenía todo moretones. (Eo1, 2020)

Mira, era eso que te tiraba en la cama y te apretaba hasta que te asfixies o te desmayabas, así era. Eso que te apretaba para no gritar, te quedabas así, después te despertabas y vos ya te despertabas ya con dolor y ves que estás amoretonada. (Eo3, 2020).

Aquí se presenta la violencia psicológica que puede ser verbal y no verbal; la primera implica insultos, burlas, ironías, desvalorizaciones, acusaciones sin razón, humillaciones en privado o en público, amenazas de muerte u otro daño a la propia persona o a los seres queridos, prohibiciones de salir de la casa, tener contacto con su familia o amistades. La 
segunda -la no verbal- supone un trato hostil, amenazante, indiferente ante las necesidades o sentimientos de la víctima, acechar los movimientos y conversaciones.

Él venía y me decía: que pa yo hice hoy, a donde pa me fui, con quien hablé, seguramente ya hiciste todo tu porquería, seguro ya estuviste con otra persona, así. (Eo1, 2020).

Era muy celoso, exageradamente celoso, por cualquier cosa me decía que yo le estaba mirando a ese, no que esto... yo prácticamente tenía que vivir encerrada en mi pieza. Él se iba a trabajar para eso nomás para que yo no salga. (E05, 2020)

No me pega, pero che tratavaieterei lo mimo voi que che enzoqueta peteĩ tongo porque una cosa mentira pues, che acusa rei. $\mathrm{Si}$, que yo soy una puta que yo tengo mucho gaucho, nambre asi me trataba" (No me pega, pero me trata muy mal, es lo mismo que me den un puñetazo, porque me acusa sin fundamentos, me decía que era una puta que tenía muchas parejas). (Eo6, 2020)

En lo que concierne a la violencia sexual que es cuando se obliga a la víctima por medio de violencia física, amenazas o chantajes a mantener cualquier actividad sexual con el agresor $u$ otras personas con o sin cobro de por medio. Someter a la víctima a tocamientos o posturas sexuales que ésta percibe como denigrantes o inadecuadas $u$ obligarla a embarazarse o abortar.

Algunas expresiones de la violencia sexual que sufrieron las mujeres entrevistadas:

Porque él vino una noche así agresivo ahí en RI, sarambí ko me hizo, me desgarró todo, fue un desgarro, desastre me hizo a raíz de eso me quedé embarazada. (Eo4, 2020).

Él [mi esposo] estaba, él vivía acá bajo la comodidad que yo puse y bajo la comodidad que ese señor me ponía [su relación paralela consentida por el esposo a cambio de beneficios materiales] y yo le culpaba a él le dije; vos me prostituiste porque una manera de prostitución fue porque no fue porque yo estaba enamorada del tipo que yo estuve con él, fue eso, y eso no le puedo disculpar no le puedo perdonar. (Eo3, 2020)

Tal cual refieren las mujeres, la introducción de la violencia fue progresiva; la dinámica incluyó el aumento de la gravedad de los hechos y las formas de la misma:

Cuando yo sabía que era su hora [de llegada del trabajo, yo] corría todo, le ponía todo [todas las cosas de la casa en orden] para que no le toque, para que no le pegue, para que no le haga nada, luego [a mis hijos]. (Eo5, 2020)

La violencia no solo ocurre en el momento de la agresión como tal, como lo indica (Vera, 2009) previo a la acción ya existen consecuencias a nivel psicológico; ocurre un agotamiento emocional que llega a veces al suicidio. En otros casos se presentan: depresión, ansiedad, fatiga crónica, pesadillas, trastornos en la alimentación, consumo de alcohol o drogas, etc.

Los sábados cobraba y ya venía borracho, yo me sentía intranquila, no sabía que iba pasar, si iba venir bien, si va ver algún problema, si íbamos a estar bien, ya estaba calculando todo ya. (E05, 2020)

Pero cada vez, cuando llega esa hora, me sentía angustiada, no quería que llegue la hora, y así. Ya cabizbaja luego, cuando él viene, termina ya la alegría. No se escucha radio, no se pone esto, todo lo que él dice nomás se hace, se come, se todo. Así era. Y más subió así su ego, vamos a decir, cuando él empezó a trabajar bien. (E04, 2020).

La típica familia nuclear -padre, 
madre e hijos, como indica (eSalud 2019), fue considerada durante mucho tiempo por la sociedad como el ideal para criar a los niños y niñas.

Ha che membykuña rehehape po no osẽ vai orehegui" (Por mis hijos, para que no sean malas personas). (Eo6, 2020)

Y yo dije, yo nunca tuve mamá ni papá, yo no quiero que mis hijos pasen eso. Yo quiero que ellos digan mamá y papá. Pero había sido que era totalmente al revés lo que estaba haciendo. Yo le estaba perjudicando a ellos psicológicamente, todo lo que ellos veían, las veces que su papá me pegaba, ellos se asustaban, lloraban. (E02, 2020)

El poder y la dominación del hombre sobre la mujer y los hijos están asociados al sistema patriarcal que legitiman el comportamiento violento para mantener así el control sobre ellos.

A parte de las mujeres también los hijos e hijas en condición de testigos de violencias son directamente afectados.

En nuestra pieza. Ellos prácticamente le tenían miedo, miedo, pero miedo le tenían a su papá. Temblaban luego cuando le veían. (Eo4, 2020).

Hubo una pelea que le pegó a mi hija, se metió la otra, me metí yo y a las tres nos pegó entonces era como que nosotros íbamos a matarnos todos acá y él no se iba ir de la casa. (Eo3, 2020)

Los hijos e hijas de las mujeres entrevistadas no solo testificaban la violencia, sino que también fueron víctimas de agresiones físicas y ambos tipos de violencia a la par tuvieron consecuencias psicológicas que desde la percepción de las madres se manifestaba de las siguientes formas:

Yo creo que se sienten mal, pero no pueden hacer nada porque le tienen miedo y 3 (tres) de ellos tienen problema de aprendizaje, y ahora sinceramente toditos están aplazados. Y los más grande lo que me dicen luego las cosas y se vuelven rebeldes." (E01, 2020).

"Y ellos crecieron traumados, esos 3 están totalmente traumados. Gracias a Dios, estos 2 últimos, ya no pasaron." (E04, 2020).

En esta misma línea las mujeres entrevistadas comentan sus experiencias judiciales;

Una noche si hice la denuncia y vino el policía y nos habló y nos dijo que la familia y que esto y aquello y acá nomás, nunca le dijeron retírate de acá o sea no tenía yo protección. (Eo3, 2020).

Y hacía la denuncia, nunca se podía hacer nada porque no tenía familiares, no tenía nadie que me ayude. Siempre ellos eran muy prepotentes. (Eo2, 2020).

Él tiene orden de alejamiento, no se cumple eso. (Eo1, 2020).

Es así que el cumplimiento de las leyes tropieza con obstáculos de diversa índole como la falta de perspectiva de género a la hora de impartir justicia, por parte de los órganos públicos responsables de operar el sistema como la Policía Nacional, el Ministerio Público y los Juzgados de Paz.

\section{Discusión y conclusiones}

La presente investigación basada en las experiencias de las mujeres madres víctimas de violencia de género que asisten al servicio comunitario Casa de la Mujer, se concluye tres aspectos:

Por un lado, como menciona Jelin (2005) la violencia resulta transgeneracional, teniendo en cuenta que las mujeres entrevistadas víctimas de violencia coinciden que todas han pasado por situaciones de violencia en sus familias, con sus madres. Por tanto, los niños, niñas y adolescentes testigos de violencia pueden volver a ser víctimas de la misma violencia 
Carosini Miranda, A. A., \& Morales Ruiz, L. P. Características socio-económicas de las familias de mujeres víctimas de violencia de género con hijos/hijas testigos, Bañado Tacumbú, 2020.

en su vida adulta si no reciben una atención integral especializada que les permita comprender las relaciones afectivas en el marco del bienestar psicológico, físico y social.

Por otro lado, los datos ponen de manifiesto que la violencia de género tiene consecuencias a largo plazo que no acaban con el solo hecho de separase del agresor, en el caso de las mujeres entrevistadas; durante la dinámica familiar dentro de un entorno violento se las ha arrebatado el derecho a la formación académica por medio del aislamiento forzoso por parte de sus parejas, la misma prohibición para insertarse en el ámbito laboral, estando así condicionadas únicamente a realizar las tareas del hogar y dependiendo económicamente del agresor.

Entre los datos recabados predomina la violencia física, psicológica y verbal, así como también hubo manifestaciones de violencia sexual hacia las entrevistadas por parte de sus ex parejas. Se percibe además el nivel de agresividad y el riesgo de muerte en que las mujeres se encontraban todo el tiempo.

Los hijos e hijas testificaron toda esta violencia y en algunos casos también fueron víctimas de las agresiones físicas. Todo esto, al igual que en sus madres, tuvo una afectación emocional lo que condicionó el rendimiento escolar, la relación interpersonal y la relación afectiva entre madre e hijo/a ya que dentro de su entendimiento los mismos responsabilizaban a sus madres por lo que estaban pasando.

En los relatos de las entrevistadas se percibe dos factores de riesgo para la permanencia dentro del círculo de la violencia; la primera expuesta por eSalud (2019), la carga cultural por pertenecer a una familia nuclear, y la segunda la percepción acerca de uno de los mitos que refiere (ComVo Mujer, 2014); la relación entre el consumo del alcohol y la violencia.

Además de todo esto, las mujeres de sectores vulnerables se encuentran con muchos obstáculos en el momento deacudir a instituciones judiciales, la intervención se limita a redactar la denuncia, pero ya no realizan ningún seguimiento ni una derivación para la atención integral de la familia.

La investigación da énfasis a la importancia de las intervenciones con perspectiva de género, el único modo de atender las necesidades de las mujeres en todas sus dimensiones como: mujer, madre, jefa de hogar, trabajadora, y sobre todo una persona con derechos.

Es importante destacar que en base a la revisión bibliográfica los resultados obtenidos muestran una escasa producción científica generada en Trabajo Social referente a las políticas sociales dirigidas específicamente a mujeres víctimas de violencia de género en sectores vulnerables y una presencia casi inexistente de la temática especifica de los niños, niñas y adolescentes como víctimas.

La violencia de género ha sido objeto de numerosos esfuerzos legislativos, técnicos e institucionales para su detección y erradicación. Sin embargo, los hijos de las mujeres víctimas han quedado relegados a un segundo plano a pesar de ser también víctimas de esta violencia.

Mientras no exista una conciencia y voluntad política que garanticen los derechos y una vida libre de violencias para las mujeres y sus hijos e hijas, todas las ratificaciones y acuerdos internacionales asumidas serán netamente un discurso, letra muerta y un mero cumplimiento diplomático lo que atribuye la impunidad de los agresores y consiente la violencia convirtiéndola en una acción natural.

\section{Referencias}

Arrom, C., Fresco, M. P., Samudio, M., Arrom, C. M., Arce, A., Arrom, M. A. (2015). Síndrome depresivo en la adolescencia asociado a género, abuso sexual, violencia física y violencia. Mem. Inst. Investig. Cienc. Salud., 13(3), 45-50.

Dirección General de Estadísticas, Encuestas y Censo (DGEEC). (2012). Asunción Bañado Sur. Principales 
indicadores de población y vivienda 2012

Dirección General de Estadísticas, Encuestas y Censo (DGEEC). (2016). Mujeres Jefas de Hogar. Periodo 20022012

Dobrée, P. (2018). ¿Cómo se las arreglan para sostener la vida? Prácticas, experiencias y significados del cuidado entre mujeres pobres que viven en el Bañado Sur de Asunción. Paraguay.

Dobrée, P. (Marzo de 2020). Cuidados ejercidos por las mujeres en el contexto covid. Asunción, Paraguay.

Jelin,E.(2005).Lasfamiliaslatinoamericanas en el marco de las transformaciones globales. Hacia una nueva agenda de políticas públicas. dds.cepal.org/ eventos/presentaciones/2005/0628/ Elizabeth_Jelin.pdf
Ministerio de la Mujer (2020). Paraguay protege de la violencia a mujeres, niñas, niños y adolescentes. Recuperado de:

Ministerio de Mujer (2019). ¡Emergencia Basta de Feminicidios. http://www. mujer.gov.py/index.php/campanas/ violencia-cero

ONU Mujeres (2016). Violencia contra las Mujeres en Paraguay: avances y desafíos. AGR S.A Servicios Gráficos.

UNICEF. (2012). Observatorio de los Derechos de la Infancia y la Adolescencia en Uruguay 2012. http:// www2.compromisoeducativo.edu.uy/ sitio/wp-content/uploads/2014/05/ Observatorio_infancia_2012.pdf

\section{Sobre las Autoras}

Analía Roxana Carosini Miranda

Licenciada en Ciencias de la Educación con Énfasis en Evaluación del Aprendizaje por la Facultad de Filosofía de la Universidad Nacional de Asunción. Licenciada en Trabajo Social por la Facultad de Ciencias Sociales de la Universidad Nacional de Asunción.

Lilian Paola Morales Ruiz

Licenciada en Trabajo Social por la Facultad de Ciencias Sociales de la Universidad Nacional de Asunción. 